\title{
Recent advances in linear series and Newton-Okounkov bodies
}

\author{
Ciro Ciliberto $^{1}$ - Alex Küronya ${ }^{2}$. Victor Lozovanu ${ }^{3}$. \\ Stefano Urbinati ${ }^{4}$
}

Published online: 27 June 2017

(C) Springer-Verlag Italia 2017

This volume is an issue of the Rendiconti del Circolo Matematico di Palermo connected to the workshop "Recent advances in Linear series and Newton-Okounkov bodies" which took place at the Department of Mathematics of the University of Padova, Italy, in February, 9-14, 2015. We thank the Editors of the Rendiconti for having accepted to dedicate this issue to this topic.

The workshop was organized by Ciro Ciliberto, Alex Küronya, Victor Lozovanu and Stefano Urbinati with the support of Foundation Compositio Mathematica, INDAM-GNSAGA, GRIFGA, Università degli Studi di Padova and Università degli Studi di Roma "Tor Vergata" and the National Research Project (PRIN) "Geometry of Algebraic Varieties".

The aim of the workshop was to bring together experts in the field of linear systems, Newton-Okounkov bodies and Nagata Conjecture to have a comparison of production in different research directions. There have been about 50 participants, both experts and young researchers in Algebraic Geometry, joining the workshop from all around the world.

The structure of the workshop was very dynamic. There have been a number of introductory talks in the mornings, whose goal was to stimulate further discussions, combined with some more advanced talks dedicated to more recent advances. In the afternoons there has

\footnotetext{
Ciro Ciliberto

cilibert@axp.mat.uniroma2.it

Alex Küronya

kuronya@math.uni-frankfurt.de

Victor Lozovanu

lozovanu@math.uni-hannover.de

Stefano Urbinati

urbinati.st@gmail.com

1 Dipartimento di Matematica, Università di Roma Tor Vergata, Roma, Italy

2 Goethe-Universität Frankfurt am Main, Institut für Mathematik, Frankfurt am Main, Germany

3 Institut für Algebraische Geometrie, Leibniz Universität Hannover, Hannover, Germany

4 Dipartimento di Matematica, Università di Padova, Padova, Italy
} 
been a division into working groups on problems, topics and issues chosen by the participants themselves.

The workshop was very successful and this volume contains some of the products of the working groups, joint with more introductory expositions. All the papers underwent a rigorous refereeing process. The contents are the following:

- C. Ciliberto, M. Farnik, A. Küronya, V. Lozovanu, J. Roé, and C. Shramov, NewtonOkounkov bodies sprouting on the valuative tree. In this paper the authors give a description of all Newton-Okounkov bodies of a big divisor on a surface with respect to valuations of the field of rational functions centered at a point. This is a research paper, which is however self-contained, i.e., it contains also short introductions to essential basic tools.

- C. Ciliberto, A. L. Knutsen, J. Lesieutre, V. Lozovanu, R. Miranda, Y. Mustopa, and D. Testa, A few questions about curves on surfaces. In this note the authors study the connection of positivity properties of a divisor on a surface in connection with the properties of being effective or movable. A basic question is treated here is: given a divisor on a surface satisfying some sort of positivity hypothesis, is there some multiple of it depending only on the surface, which is effective or movable? The authors describe some examples, discuss some conjectures and prove some results that suggest that the answer to the above question should in general be negative, unless one makes some really strong assumptions.

- O. Dumitrescu, E. Postinghel and S. Urbinati, Cones of effective divisors on the blown-up $\mathbb{P}^{3}$ in general lines. The authors compute the facets of the effective cones of divisors on the blow-up of $\mathbb{P}^{3}$ along lines in general position. They prove that up to six lines these threefolds are weak Fano and hence Mori Dream Spaces.

- C. Maclean, A review of geometrically defined functions on Newton-Okounkov bodies. In this note the author gives a presentation of two functions carrying geometric information, recently defined on Newton-Okounkov bodies by Sebastien Boucksom and Huayi Chen on one side and Daivd Witt Nystrom in the other.

- T. Szemberg, J. Szpond, On the containment problem. In this note, the authors provide an overview of the containment problem for symbolic and ordinary powers of homogeneous ideals, related conjectures and examples, focusing on ideals with zero dimensional support.

- S. Urbinati, On the ampleness and bigness of non-integral divisors. The author studies which of the classical characterizations of ample and big divisors can be extended to non-integral divisors via the corresponding line bundles. 